\title{
Interactive comment on "Soil Atterberg limits of different weathering profiles of the collapsing gullies in the hilly granitic region of south China" by Yusong Deng et al.
}

\section{Anonymous Referee \#4}

Received and published: 4 January 2017

\section{General comment:}

The study falls within the scope of Solid Earth. The paper is interesting, but it is not performed to be well understood. There are numerous amendments and explanations required. The English is not understandable in many places. The manuscript is too wordy and contain repeated facts already previously mentioned (see specific comments). Also, there is lack of novelty of this study. Although phenomenon of collapsing gully deserves better explanation of indicators, present study does not explain it. This is main fault of the paper. Without comparison of soil properties with close surrounding soil not affected with by collaps, present paper only repeats well known facts of correlation of Atterberg limits with presented soil properties. When updating, please compare 
your results with surrounding soil. Also, take more deep insight in relation: type of clay vs. dominating exchangeable cations (in CEC). This can help to explain better the results of plastic and liquid limit in each location. Some statements and conclusions are well known from earlier literature, while part of section are beyond the limits of the results (conclusion in lines 519-525). For these reasons I can not recommend this paper for publication in this condition.

Some specific comments are below:

Line 37-42: I notice that authors use long sentences. In many occasions long sentences make paper difficult to follow. Please rather use shorter and direct statements. Line 47: ". . leading to a loss of bottom soil layer..." Unclear, please rewrite. Line 6265: This statement is unclear. What are cited authors find? This is example of absence of continuity in paper. In several occasions I missed the point I was following. Line 6567: Please add word "recommended". This sentence can mean that you cannot till wet soil. It is not recommended if you want to keep your soil structural stability. Line 73-75: Please state sources in end of sentence. Line 77-79: Avoid general statements. It is better to state an important finding of each study you cite. Also, please avoid adjectives like "mountain soils". Soils have their own characteristics. Line 80-82: Please recheck English again. Line 98-100: Unclear sentence. Please rewrite. Line 105-110: Authors repeat again previously mentioned facts. Delete it. Line 119: Define "granite soil". Line 125-126: unify words, eg. weathering layer - upper soil layers. Use "topsoil" and "subsoil layers" instead. Line 131-133: Please delete "The liquid limit and plastic limit of soil, namely..." It is already mentioned before. Line 133-135: Authors write general statement. Please avoid this. Great part of Introduction authors explain the influence of water content on collapsing gully, but here is not mentioned the existing research about relation of Attenberg limits vs. soils characteristics in hilly southern China. Please write the papers who studied similar problem and their key findings. Here you can explain the novelty of your paper. Line 136-137: this goes in Materials and methods section. Line 155: Please find a better word than "serious". Line 164-166. Repeating informa-

Interactive comment
Printer-friendly version

Discussion paper 
tions mentioned before. Please delete. Line 167-168: It is unclear how many samples authors have: per layer, per location and in total. Please state this informations. Line 194-200. Did authors use appropriate statistical test? Is your data normal? Please test it and state the test and $p$ value. Also, authors in whole manuscript repeat "regressions" but I can find only correlations between paired soil characteristics. Authors can also test regressions between 3 and more data and see the nature of their connections. Line 212-214: This is huge shortcoming. Please upload a statistics in Figure 2, 4 and 5. Line 2014-2016: This is speculation. This paper did present aggregate distribution and humus content. Add source that confirm this finding or delete it. Line 216-218: Content of iron oxide does not correspond with highest PD at each location. Line 284-288: This is contrary with explanation of BD in line 233-235. Line 295-297: Missing statistics in Fig 4 and 5. Line 332-335: Do not repeat facts mentioned before (methods section).Please delete it. Line 343 and 356: Please, add letter beyond number of figure. Line 362: Change number into reference. Line 362-363: Which soil properties? Can authors draw a comparison with their own results? Line 363-367: Instead the authors explain again what plasticity index mean, it is better to explain their relationship with other soil properties and try to compare them with different studies. Line 396: Please define "external erosion" Line 428: Change Table 4 into Table 5 Line 433-437: Authors stated the significant relationships, although some of them are very weak. Please recheck your statistics again.

Sections 3.3.: Sections that contain correlations between variables are well known fact previously published (eg. particle size distributions vs Attenberg limits). Instead try to find differences between collapsing gullies and surrounding soils and then find a "go home message". Line 500-506: Delete it. Conclusions: Rewrite according the suggestions from general comments. Figures: Please delete figure 6 . The same data exist in table 5. Add statistics in tables 2, 4 and 5. Define relationship between variables used for correlations in Materials and Methods section. Tables: Add $p$ value in table 5. 